\title{
Abstract from Current Literature
}

\section{$C$ reactive protein in healthy term newborns during the first $\mathbf{4 8}$ hours of life}

Serafina Perrone, Federica Lotti, Mariangela Longini, Annalisa Rossetti, Ilaria Bindi, Francesco Bazzini, Elisa Belvisi,PasqualeSarnacchiaro, Carlo Scapellato, Giuseppe Buonocore

Archives of Disease in Childhood - Fetal and Neonatal Edition 2018; 103 F91-F91 Published Online First: 19 Feb 2018. doi:10.1136/archdischild2018-314891

Background Early-onset neonatal sepsis (EOS) is a serious and potentially life-threatening disease in newborns. $C$ reactive protein (CRP) is the most used laboratory biomarker for the detection of EOS. Little is known about normal reference values of CRP during the perinatal period as several factors are able to influence it.

Objectives To identify an appropriate range of CRP values in healthy term newborns during the first 48 hours of life.

Design CRP determination was performed in 859 term newborns at 12, 24 and 48 hours of life. Mode of delivery, maternal vaginal culture results, intrapartum antimicrobial prophylaxis (IAP) and other perinatal variables were recorded.

Results CRP mean values were significantly higher at 48 hours $(4.10 \mathrm{mg} / \mathrm{L})$ than at both $24(2.30 \mathrm{mg} /$ $\mathrm{L}$ ) and 12 hours of life ( $0.80 \mathrm{mg} / \mathrm{L})$. CRP levels were affected by a number of perinatal proinflammatory variables. In particular, CRP mean values were significantly higher in babies born by vaginal delivery $(3.80 \mathrm{mg} / \mathrm{L})$ and emergency caesarean section (3.60 $\mathrm{mg} / \mathrm{L}$ ) than in babies born by elective caesarean section $(2.10 \mathrm{mg} / \mathrm{L})$. Completed course of IAP led to lower CRP mean values $(2.90 \mathrm{mg} / \mathrm{L})$ than IAP not completed (3.80 mg/L) or not performed $(4.7 \mathrm{mg} / \mathrm{L})$.

Conclusions Postnatal age and mode of delivery significantly influence CRP values. Reliable reference values are crucial in order to obtain an adequate diagnostic accuracy.

\section{Rapid Diagnostic Tests in Childhood Infections}

RITABRATA KUNDU From Department of Pediatrics, Institute of Child Health, Kolkata, India. rkundu22 @gmail.com

INDIAN PEDIATRICS, VOLUME 55 MARCH 15, 2018

Presumptive treatment of infections often results in irrational antimicrobial use resulting in detrimental spread of drug resistance and untoward side effects. A rapid diagnostic test (RDT) is a test that delivers a result earlier than conventional testing methods employed in the past to identify the offending microorganism. RDTs help in early definitive therapy, reduction in hospital stay and cost, and in degree of morbidity and mortality associated with the infection. To select a proper RDT, one should consider how specific and sensitive the test is. Most RDTs gives a qualitative result not quantitative; hence disease severity, monitoring of the disease, prognostication and therapeutic efficacy cannot be assessed. A RDT should be easy to perform, should not require sophisticated machines, and kits should be stable in extremes of temperature. RDTs may be of immense help in remote places where conventional diagnostic facilities are unavailable or lack quality. RDTs hold promise of reasonable diagnostic accuracy if done in a optimal clinical background. They should never be ordered as a shotgun approach to exclude all possible infections but should be used judiciously with appropriate interpretation. Keywords: Diagnosis, Dengue, Tuberculosis, Typhoid, Serology, Scrub typhus.

KEY MESSAGES: - Rapid diagnostic tests ensure definitive diagnosis of infections, thereby preventing unnecessary presumptive treatment. - These usually provide qualitative results and are not useful in monitoring treatment.

- These tests require minimum skill making them useful in places without sophisticated laboratory infrastructure. - These tests must always be ordered on the basis of clinical setting, and not as a battery. 
Efficacy, cost effectiveness, and sustainability of a pediatric high risk asthma clinic

Nadia L. Krupp, Andrea Weist, Cindy D. Fiscus, James Slaven, Andrew Harner, Gregory S. Montgomery, Michelle S. Howenstine

Pediatric Pulmonology. May 2018; Volume 53, Issue 5: Pages 538-543

https://doi.org/10.1002/ppul.23967

Aim: At our institution, a pediatric High Risk Asthma clinic has been in operation for over 15 years, targeting children with poorly controlled, and difficult to treat asthma. This study evaluates the outcomes and cost effectiveness of the High Risk Asthma clinic from 2000 through 2014.

Methods: A retrospective chart review was performed on all patients entering High Risk Asthma clinic from
2000 2014, and total hospitalizations and Emergency Department visits were tallied for the year prior to entering clinic and the year after. Costs incurred, and reimbursements obtained from payors were tallied to determine cost effectiveness and sustainability.

Results: Consistent decreases in hospitalizations (51.2\% decrease, $P<0.001)$ and Emergency Department visits $(23.0 \%$ decrease, $P=0.048$ ) were seen for patients entering High Risk Asthma clinic, with commensurate significant decreases in related costs. Reimbursements received for outpatient services were sufficient to offset operational costs of the High Risk Asthma clinic, when both clinic visit, and pulmonary function testing charges were included.

Conclusions: A pediatric High Risk Asthma clinic model is efficacious in decreasing hospitalizations and Emergency Department visits for a difficult to treat population, and such a model can be cost effective and sustainable. 